\title{
REVIEW
}

\section{Recent progress on the role of ChREBP in glucose and lipid metabolism}

\author{
Katsumi Iizuka1), 2) \\ ${ }^{1)}$ University Hospital Center for Nutritional Support and Infection Control, Gifu University, Gifu 501-1194, Japan \\ ${ }^{2)}$ Department of Diabetes and Endocrinology, Graduate School of Medicine, Gifu University, Gifu 501-1194, Japan
}

\begin{abstract}
Carbohydrate response element binding protein (ChREBP) is a transcription factor activated by glucose that is highly expressed in liver, pancreatic $\beta$-cells, brown and white adipose tissues, and muscle. We reported that hepatic suppression of the Chrebp gene improves hepatic steatosis, glucose intolerance, and obesity in genetically obese mice. Moreover, we have studied the role of ChREBP with special reference to feedforward and feedback looping in liver and pancreatic $\beta$-cells. Recently, several groups reported that (1) glucose activates ChREBP- $\alpha$ transactivity and in turn ChREBP- $\alpha$ induces ChREBP- $\beta$ on both transcriptional and translational levels in adipose tissues, and (2) ChREBP regulates glucose transporter type $4 \mathrm{mRNA}$ levels, which may affect glucose uptake in adipose tissues. Moreover, in adipose tissues of obese patients, Chrebpb mRNA levels were much lower than those in lean subjects, while the levels were much higher in liver of obese patients than those in lean subjects. These findings suggest that Chrebpb mRNA levels are different in various tissues and probably in the stages of diabetes mellitus. Herein, we review recent progress in the study of ChREBP with special references to (1) the mechanisms regulating ChREBP transactivity (posttranslational modifications, intramolecular glucose sensing module, feedforward mechanism, and the feedback loop between ChREBP and its target genes), and (2) the role of ChREBP in liver, pancreatic islets and adipose tissues. Understanding the role of ChREBP in each tissue will provide important insight into the pathogenesis of metabolic syndrome.
\end{abstract}

Key words: Carbohydrate response element binding protein(ChREBP), Transketolase, Krüppel like factor-10, Glucagon receptor, Basic helix-loop-helix domain containing B2/differentially expressed in chondrocytes 1 (BHLHB2/DEC1)

\section{The regulation of ChREBP transactivity}

Carbohydrate response element binding protein (ChREBP) is a basic helix-loop-helix leucine zipper transcription factor [1-3]. ChREBP and Max-like protein $\mathrm{X}(\mathrm{Mlx})$ form heterodimers $[4,5]$ and bind to the carbohydrate response element (ChoRE), which is composed of two E-boxes separated by five base pairs (CABGTG-nnCnG-nGnSTG) [6-8]. The ChREBP/Mlx heterodimer regulates glucose and lipid metabolism through regulation of the glycolytic, gluconeogenic, and lipogenic gene expression [2, 5, 8-12] (Table 1). Moreover, ChREBP regulates gene transcription, circadian rhythm, hormones and their receptors, and the

Submitted Mar. 25, 2013; Accepted Mar. 26, 2013 as EJ13-0121 Released online in J-STAGE as advance publication Apr. 19, 2013

Correspondence to: Katsumi lizuka, University Hospital Center for Nutritional Support and Infection Control, Gifu University, 1-1 Yanagido, Gifu, Gifu 501-1194, Japan.

E-mail: kiizuka@gifu-u.ac.jp redox signal [13-21] (Table 1).

Formerly, ChREBP was thought to be regulated by the classic mechanism of phosphorylation/dephosphorylation of ChREBP itself [22-24]. Recently, however, ChREBP- $\beta$, the constitutively active isoform, was identified [25]. Therefore, the mechanisms of ChREBP regulation should be revised as follows (Fig. 1):

1. Posttranslational modifications of ChREBP:

Phosphorylation/dephosphorylation, acetylation, and O-linked GlcNAcylation

2. Intramolecular glucose sensing module:

Glucose-response activation conserved element (GRACE) and low-glucose inhibitory domain (LID)

3. Feedforward control:

ChREBP isoforms (ChREBP- $\alpha$ and $-\beta$ ) and metabolites (G6P, X5P and F-2,6- $\mathrm{P}_{2}$ ) affecting ChREBP transactivities

4. Feedback control:

Crosstalk between ChREBP and its target genes 
Table 1 List of ChREBP regulated Genes

\begin{tabular}{|c|c|c|c|}
\hline Pathway & Gene (Gene Symbol) & $\begin{array}{c}\text { Glucose activation/ } \\
\text { repression }\end{array}$ & References \\
\hline \multirow[t]{4}{*}{ Glycolysis } & Liver type pyruvate kinase $(P k l r)$ & Activation & {$[1,2]$} \\
\hline & Fructokinase $(F k)$ & Activation & {$[2]$} \\
\hline & Glucose transporter 2 (Glut2) & Activation & [2] \\
\hline & Glucose transporter 4 (Glut4) & Activation & [8] \\
\hline Gluconeogenesis & Glucose 6 phosphatase catalytic subunit (G6pc) & Activation & {$[2,10,49]$} \\
\hline \multirow[t]{7}{*}{ Lipogenesis } & Fatty acid synthase (Fasn) & Activation & {$[2,5,8,49]$} \\
\hline & Acetyl coA carboxylase $1(A c c 1)$ & Activation & {$[2,5,8,49]$} \\
\hline & Stearyl coA desaturase $1(S c d 1)$ & Activation & {$[2,8,49]$} \\
\hline & ELOVL fatty acid elongase 6 (Elovlo) & Activation & {$[12]$} \\
\hline & Malic Enzyme $(M e)$ & Activation & {$[2]$} \\
\hline & Adiponutrin/Patatin-like phospholipase domain-containing protein 3 (Pnpla3) & Activation & [11] \\
\hline & Spot 14 & & [2] \\
\hline \multirow[t]{6}{*}{ Transcription } & Krüppel like factor-10 (Klf10) & Activation & {$[14]$} \\
\hline & Differentially expressed in chondrocytes 1 (Decl) & Activation & [13] \\
\hline & Pancreatic and duodenal homeobox $1(P d x l)$ & Repression & [19] \\
\hline & Peroxisome proliferator activated receptor $\alpha$ (Ppara) & Repression & [63] \\
\hline & Hypoxia inducible factor-1b (Hiflb) & Repression & {$[20]$} \\
\hline & Sirtuin $1($ Sirt 1$)$ & Repression & [17] \\
\hline \multirow[t]{4}{*}{ Hormone and its receptor } & Insulin 1 (Ins 1$)$ & Repression & [19] \\
\hline & Insulin 2 (Ins2) & Repression & [19] \\
\hline & Glucagon receptor (Gcgr) & Activation & {$[16]$} \\
\hline & Regulator of G-protein Signaling 16 ( $\left.\operatorname{Rgs}_{\mathrm{S}} \mathrm{C}\right)$ & Activation & [18] \\
\hline Redox signal & Thioredoxin interacting protein (Txnip) & Activation & [21] \\
\hline
\end{tabular}

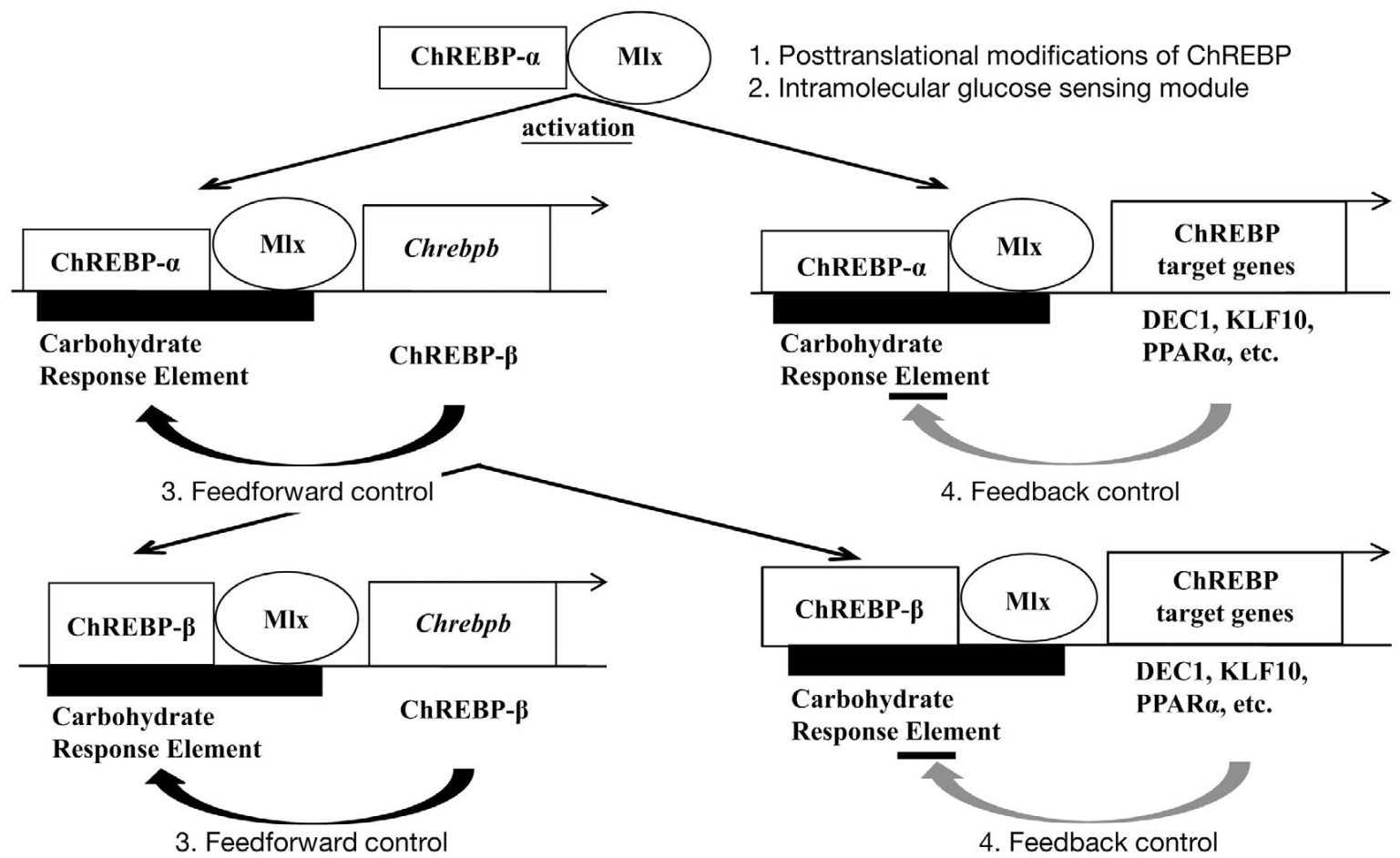

Fig. 1 Schematic representation of the mechanisms regulating ChREBP transactivities

Glucose activates ChREBP- $\alpha$ through (1) posttranslational modification of ChREBP, and (2) an intramolecular glucose sensing module. In turn, ChREBP- $\alpha$ induces ChREBP- $\beta$ (3. feedforward control). Some ChREBP target genes inhibit both ChREBP- $\alpha$ and $\beta$ transactivities (4. feedback control). 


\subsection{Posttranslational modifications of ChREBP (Phosphorylation/dephosphorylation, acetylation, and O-linked GlcNAcylation)}

\subsubsection{Phosphorylation/dephosphorylation}

The phosphorylation/dephosphorylation of ChREBP has been proposed as the classic mechanism for regulating ChREBP transactivity [22-24]. Recently, it has been reported that ChREBP has two isoforms, ChREBP- $\alpha$ and ChREBP- $\beta$, and that they are located mainly in the cytosol and nucleus, respectively [25]. After glucose stimulation, ChREBP- $\alpha$ is translocated into the nucleus and binds to ChoRE after its dephosphorylation by protein phosphorylase $2 \mathrm{~A}$, which is regulated by xylulose 5 -phosphate (X5P) in the pentose phosphate pathway [23]. Under starvation conditions, ChREBP is inactivated by phosphorylation at Ser196, Ser626 and Thr666 by cAMP-dependent protein kinase (PKA), and at Ser568 by AMP-activated protein kinase (AMPK) [22, 24]. However, another study has reported that mutation of the phosphorylation sites Ser196, Ser626 and Thr666 in ChREBP did not affect ChREBP transactivity [26]. Our group as well as other groups also confirmed that glucagon, which increases cAMP levels, inhibits the glucose-induced expression of ChREBP target genes $[16,27,28]$, but further investigation may be needed to clarify in detail the significance of the phosphorylation/ dephosphorylation mechanism.

\subsubsection{Acetylation of ChREBP}

Recently, it has been reported that histone acetyltransferase (HAT) coactivator p300 associates with ChREBP to acetylate Lys672 and promote ChREBP DNA binding [29]. ChREBP acetylation by p300 is negatively regulated by salt-inducible kinase 2 (SIK2), which is activated under malnutrition conditions [29]. In ob/ob mice, SIK2 is inhibited and consequently the binding of ChREBP to ChoRE is increased after ChREBP hyperacetylation by p300 [29]. Thus, inhibition of p300/HAT activation may be a therapeutic target for hepatic steatosis (Fig. 2).

\subsubsection{O-linked GIcNAcylation}

O-linked- $\beta-\mathrm{N}$-acetylglucosamine (O-GlcNAc) is the end product of the hexosamine biosynthesis pathway and is modified by two enzymes: O-GlcNAc transferase (OGT) and O-GlcNAcase (OGA) (Fig. 3) [30]. The O-linked- $\beta$-N-acetylglucosamine modification, O-GlcNAcylation, is a nutrition-dependent posttranslational modification. ChREBP undergoes O-GlcNAcylation, which increases its protein levels and the transactivation of its target genes [31-33]. Moreover, overexpression of FoxO1 reduces

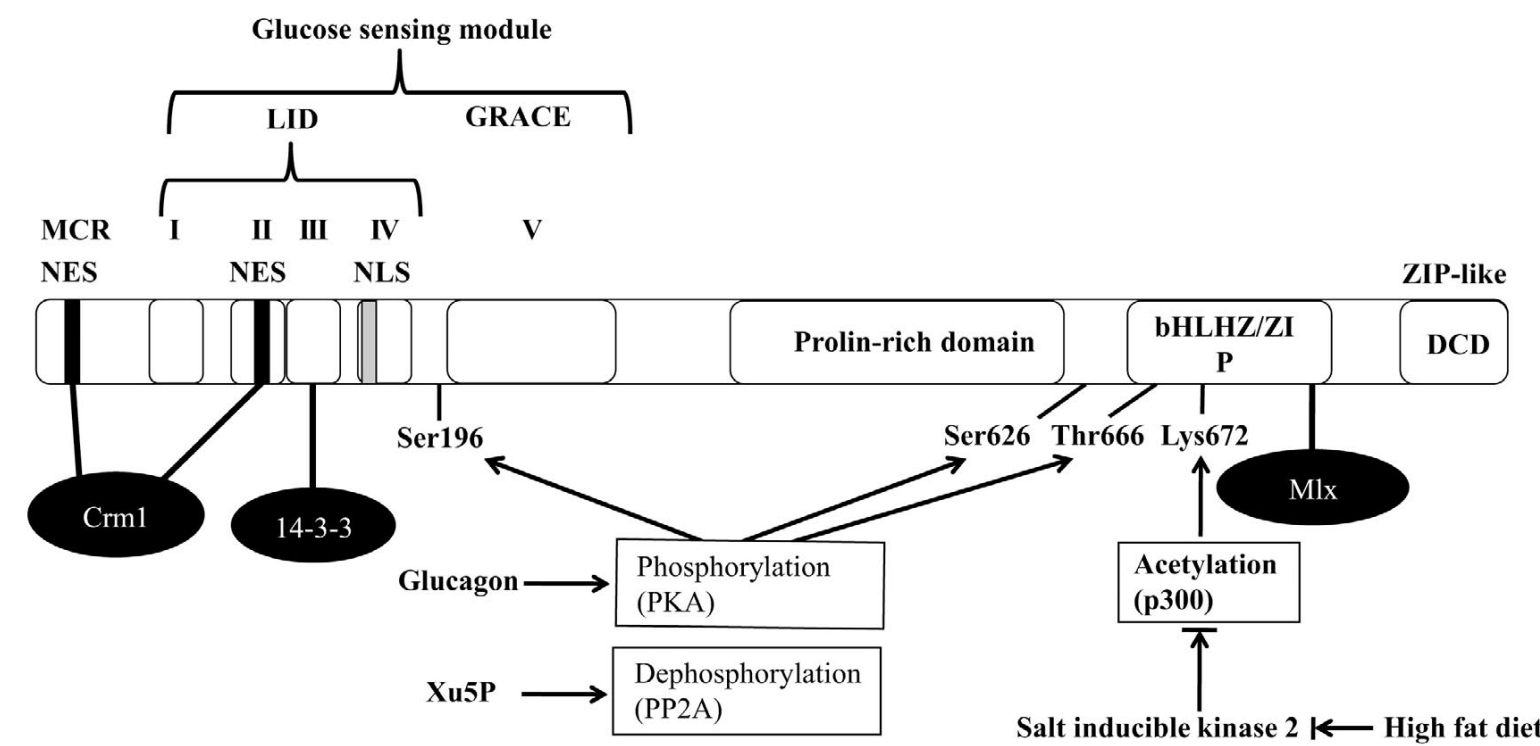

Fig. 2 Schematic representation of ChREBP- $\alpha$ protein

ChREBP- $\alpha$ contains a glucose sensing module (GSM). GSM contains a low glucose inhibitory domain (LID) and glucose response activation conserved element (GRACE). LID contains the mondo conserved region (MCR) I-IV and GRACE contains MCR V. Ser196, Ser626 and Thr666 are phosphorylation sites for cAMP-dependent protein kinase (PKA), and Lys672 is an acetylation site for histone acetyltransferase (HAT) coactivator p300. Salt inducible kinase 2 inhibits p300/HAT activity. In ob/ ob mice, Salt inducible kinase 2 is inhibited.

NES, nuclear export signal; NLS, nuclear localization signal; bHLHZ, basic helix-loop-helix leucine zipper 


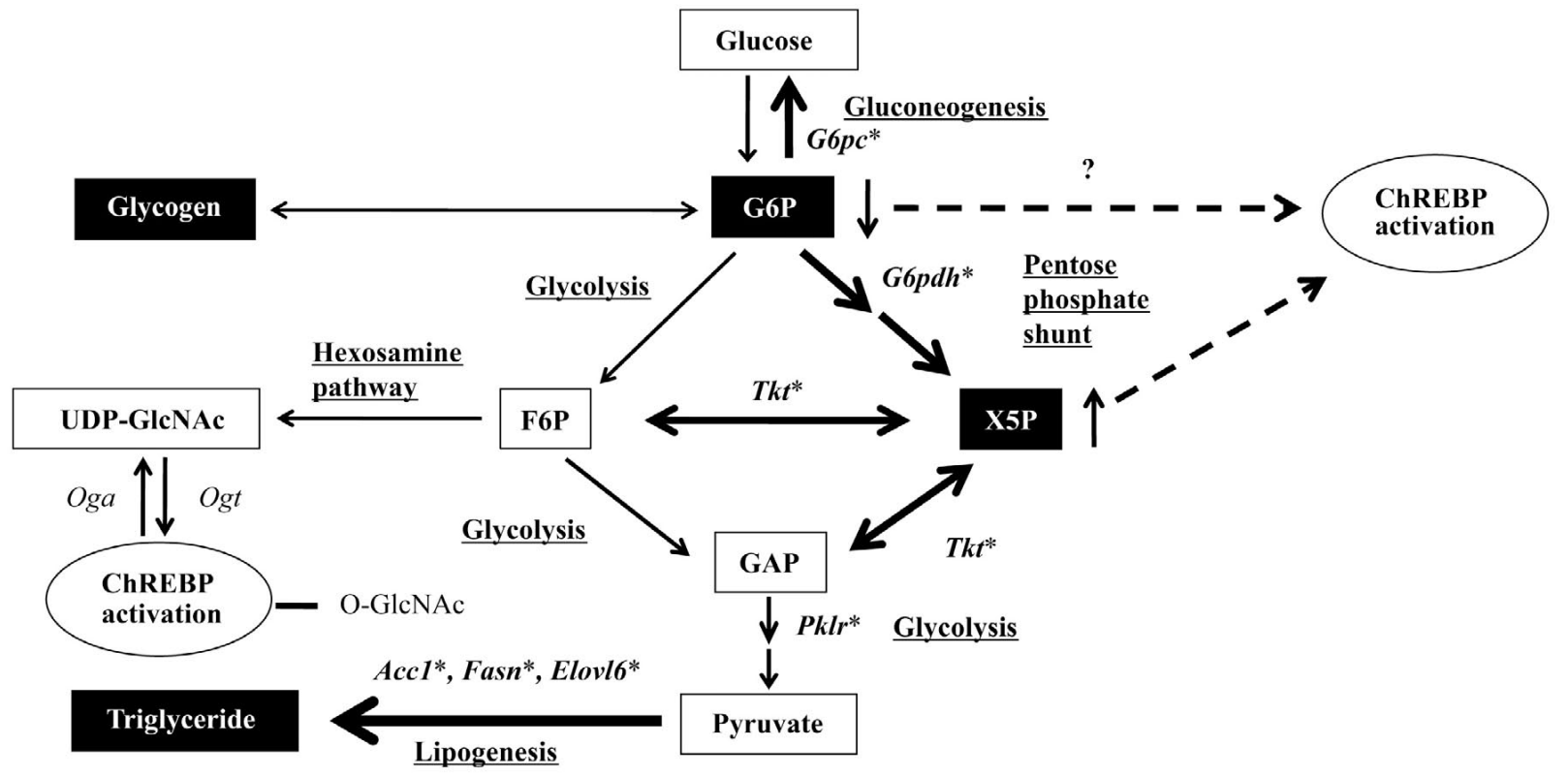

Fig. 3 Metabolites and enzymes affecting ChREBP transactivity

Glucose 6-phosphate (G6P), xylulose 5-phosphate (X5P), and fructose 2,6-bisphosphate are candidate molecules for activating ChREBP transactivity. The concentrations of G6P and X5P are regulated by ChREBP target genes such as glucose6-phosphatase, catalytic subunit (G6pc), glucose-6-phosphate dehydrogenase (G6pdh), transketolase (Tkt), and liver type pyruvate kinase ( $P k l r)$, and several lipogenic genes (Fasn, Accl, Elovl6). Moreover, O-linked- $\beta$-N-acetylglucosamine is the end product of the hexosamine biosynthesis pathway, and is modified by O-GlcNAc transferase (OGT) and O-GlcNAcase (OGA). Thus, ChREBP regulates its transactivity by regulating the expression of these genes. * indicates ChREBP target genes.

ChREBP transactivity, and gene ablation of FoxO1 increases ChREBP transactivity through enhanced O-glycosylation and increased ChREBP protein levels [33]. In view of the fact that FoxO1 activity is regulated by insulin, O-GlcNAcylation of ChREBP may play an important role in the insulin-mediated ChREBP activation [33]. Thus, posttranslational modifications of ChREBP may regulate ChREBP transactivity.

\subsection{Intramolecular glucose sensing module Iglucose- response activation conserved element (GRACE) and low-glucose inhibitory domain (LID)]}

ChREBP- $\alpha$ and $-\beta$ are composed of 864 and 687 amino acids, respectively [25]. ChREBP- $\alpha$ contains a glucose-sensing module (GSM) (Fig. 2) [34-38]. GSM contains a low-glucose inhibitory domain (LID, residues 37-192) and a glucose-response activation conserved element (GRACE, residues 197-298) (Fig. 2) [34-38]. Mondo conserved region (MCR) is composed of five distinct subdomains, MCR I-V, of which MCR I-IV corresponds to LID (Fig. 2) [34-38]. ChREBP- $\alpha$ contains a nuclear localization signal (NLS) in MCR IV and two nuclear export signals (NES1 and NES2) bound by nuclear export factor, Crm1 (Fig. 2) [34-38]. MCR III is constitutively bound by $14-3-3$, which is needed for glucose responsiveness [36]. 14-3-3 binding may also contribute to the cytoplasmic localization of ChREBP $[37,38]$. In contrast, ChREBP- $\beta$ only contains GRACE [25]. As mentioned above, ChREBP- $\alpha$ is localized in both the nucleus and cytosol, and ChREBP- $\beta$ is localized only in the nucleus. Thus, ChREBP- $\alpha$ and ChREBP- $\beta$ sense glucose concentration through different mechanisms.

\subsection{Feedforward control}

\subsubsection{ChREBP isoforms, ChREBP- $\alpha$ and $-\beta$}

As mentioned above, ChREBP has two isoforms, ChREBP- $\alpha$ and $-\beta[25,39]$. Previously, we reported that overexpression of dominant negative Mlx (dnMlx), a ChREBP antagonist, inhibits glucose induction of Chrebp mRNA expression in rat primary hepatocytes [12]. In addition, in rat primary hepatocytes, glucose increases the mRNA levels of Chrebp and transketolase $(T k t)$, which catalyzes the production of X5P, a candidate activator of ChREBP (Fig. 3) [39]. We hypothesized that there is a feedforward mechanism 
and searched for a ChoRE in the Chrebpa promoter, but we could not detect it. Moreover, Chrebpa mRNA is regulated by insulin, LXR, and thyroid hormone, but not glucose [40-42]. Recently, it has been reported that ChREBP- $\beta$, a novel isoform of ChREBP, is increased by glucose in a dose-dependent manner, while Chrebpa mRNA expression is not affected by glucose [25, 39], and that Chrebpa and Chrebpb mRNA are regulated by two different promoters [25]. Moreover, ChoRE of ChREBP- $\beta$ was found to be located near exon $1 b$ in the ChREBP target genes [25]. Using reporter analysis of pGL3 promoter vector inserted with ChoRE of ChREBP- $\beta$, we confirmed that ChoRE is also functional in rat insulinoma-derived INS-1E cells [39]. Chrebpb mRNA expression is less abundant than Chrebpa, but ChREBP- $\beta$ is a more potent transactivator at both low and high glucose concentrations [25]. Thus, these findings further indicate that ChREBP- $\alpha$ and ChREBP- $\beta$ sense glucose levels through different mechanisms.

\subsubsection{Metabolites affecting ChREBP transactivity}

What kind of metabolite activates ChREBP transactivity has been controversial (Fig. 3) [23, 39, 43-47]. In particular, X5P, glucose 6-phosphate (G6P) and fructose-2,6-bisphophate (Fru-2,6- $\mathrm{P}_{2}$ ) are candidate molecules for regulating the expression of ChREBP target genes (Fig. 3) [23, 39, 43-47]. Kabashima et al. reported that $\mathrm{X} 5 \mathrm{P}$ activates protein phosphatase $2 \mathrm{~A}$ to activate ChREBP transactivity [23]. Recently, it has been reported that both G6P and Fru-2,6- $\mathrm{P}_{2}$ activates ChREBP transactivity [45-47]. We tested the effect of G6P and X5P on ChREBP transactivity; however, the activities induced by G6P and X5P were lower than the glucose-induced activity [39]. These findings suggest that the mechanism by which glucose activates the transactivity of ChREBP is very complicated and further investigation is required to clarify this mechanism.

1.3.3 Enzymes affecting G6P and X5P (Glucose-6phosphatase catalytic subunit and transketolase)

Glucose-6-phosphatase, catalytic subunit (G6PC) is a gluconeogenic enzyme that converts G6P into glucose [48]. G6PC is expressed in limited tissues such as liver and kidney; however, it is not expressed in pancreatic $\beta$-cells and adipose tissues [48]. In view of the fact that G6PC activity determines the G6P concentration, the role of G6PC in the regulation of ChREBP transactivity may be limited to the liver and kidney. We have reported that hepatic G6pc mRNA levels in Chrebp ${ }^{-/-}$ mice and ob/ob Chrebp ${ }^{-/-}$mice were much lower than in WT and ob/ob mice, respectively [2, 49]. Moreover,
Pedersen et al. identified that the ChoRE in the rat G6pc promoter is located between -3706 and -3682 bp [10]. Together with the fact that G6Pase converts G6P to glucose, these findings suggest that ChREBP and G6Pase may form a feedback loop (Fig. 3).

$\mathrm{X} 5 \mathrm{P}$ is an intermediate molecule in the pentose phosphate pathway $[12,23,39]$. Transketolase (TKT) is the enzyme that produces X5P from two glycolytic intermediates, glyceraldehyde 3-P (GAP) and fructose-6-P (F6P) (GAP + F6P $\leftrightarrow$ X5P + erythrose 4-P). X5P activates protein phosphatase $2 \mathrm{~A}(\mathrm{PP} 2 \mathrm{~A})$, which in turn activates ChREBP by dephosphorylation [23]. Thus, TKT connects the pentose phosphate pathway with the glycolytic pathways. Tkt mRNA is listed in groups of glucose-induced genes [8, 12]. We have demonstrated that $T k t$ mRNA is induced by glucose in a dose-dependent manner, and that this induction is induced by adenoviral overexpression of ChREBP [12]. Moreover, Chrebp mRNA is induced by glucose in a dose-dependent manner, corresponding with Tkt mRNA [12]. Together with the fact that X5P is a potential activator of ChREBP, TKT and ChREBP constitute a feedback loop that regulates ChREBP transactivity (Fig. 2) [12].

\subsection{Feedback control (Crosstalk between ChREBP and its target genes)}

It is known that the activity of transcription factors is regulated by their target molecules. For example, circadian rhythm consists of a network of transcriptional-translational feedback loops that drive rhythmic, 24-h expression patterns of core clock components [50]. In the primary feedback loop, the positive elements include members of the basic helix-loop-helix (bHLH)-PAS (Period-Arnt-Single-minded) transcription factor family, CLOCK and BMAL1. CLOCK and BMAL1 heterodimerize and initiate transcription of target genes containing E-box cis-regulatory enhancer sequences, including Period genes (in mice, Per 1, Per 2 and Per3) and Cryptochrome genes (Cryl and Cry2) [50]. Negative feedback is achieved by PER:CRY heterodimers that translocate back to the nucleus to repress their own transcription by acting on the CLOCK:BMAL1 complex [50]. According to the concept that ChREBP is directly and indirectly repressed by its target molecules, we identified the feedback loop between ChREBP and its target molecules (e.g., DEC1, KLF10 and PPAR $\alpha$ ) (Fig. 4) [13-16]. 


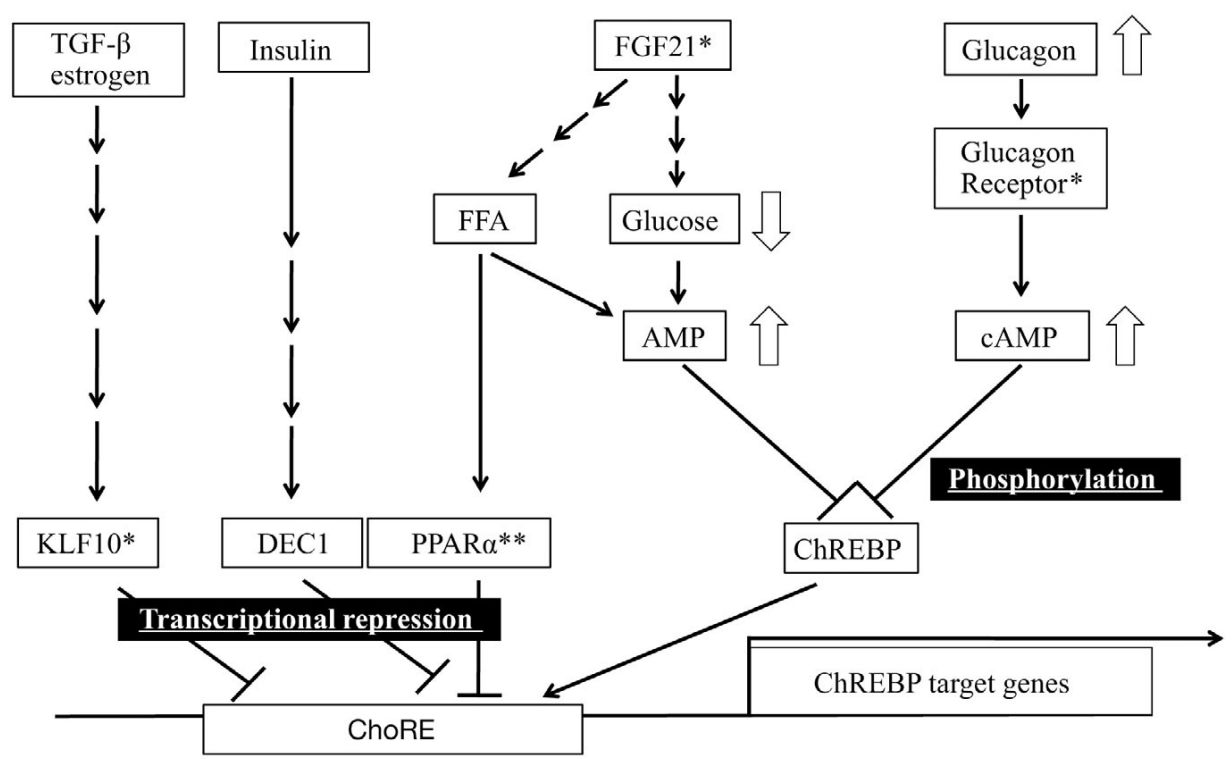

Fig. 4 Feedback loop between ChREBP and ChREBP target genes

The feedback systems of ChREBP regulation are composed of two factors: 1. Transcription factors (KLF10, DEC1 and $\operatorname{PPAR} \alpha$ ), and 2. hormone, hormone receptor and signal transduction (Glucagon receptor, Fibroblast growth factor-21).

* indicates ChREBP target genes. ** indicates gene suppressed by ChREBP.

1.4.1 Basic helix-loop-helix domain containing, class B, 2/ differentially expressed in chondrocytes 1 (Bhlhb2/Dec1)

Basic helix-loop-helix domain containing, class B, 2/ differentially expressed in chondrocytes 1 (BHLHB2/ DEC1) is also known to play an important role in the regulation of the mammalian circadian rhythm [51, 52]. Recently, it has been reported that deletion of the Clock gene causes metabolic syndrome, and that Bhlhb2 mRNA is increased in muscles of diabetic and insulin-resistant humans [53, 54]. These findings suggest that BHLHB2 plays an important role in the development of metabolic syndrome. However, its precise role in metabolism remains unclear. We investigated the link between BHLHB2 and ChREBP, a glucoseactivated transcription factor involved in the regulation of lipogenesis. Glucose stimulation and overexpression of dominant active ChREBP induced Bhlhb2 mRNA expression in rat hepatocytes [13]. Deletion studies have shown that ChoRE ( -160 to $-143 \mathrm{bp}$ ) in the mouse Bhlhb2 promoter region is functional in vivo [13]. Overexpression of BHLHB2 inhibits glucose and ChREBP-mediated induction of rat fatty acid synthase (Fasn) and liver pyruvate kinase (Pklr) mRNA. Chromatin immunoprecipitation (ChIP) analysis demonstrated that Bhlhb2 binds to ChoRE in the Fasn, Pklr and Bhlhb2 promoter regions in vivo [13]. In conclu- sion, BHLHB2 and ChREBP constitute a novel feedback loop involved in the regulation of lipogenesis (Fig. 3). Recently, it has been demonstrated that BHLHB2 suppresses the transactivities of SREBP1 (sterol regulatory element-binding protein 1) and RXR (retinoid X receptor) $[55,56]$. Thus, DEC1 is a potential important regulator of the coordination of glucose and lipid metabolism.

\subsubsection{Krüppel-like factor-10 (KLF10).}

Krüppel-like factors (KLFs) are zinc finger-containing transcription factors that regulate proliferation, differentiation, development, and programmed cell death [57]. KLF10 was initially identified as a primary transforming growth factor (TGF)- $\beta$-inducible early gene in human osteoblasts [57]. KLF10 and KLF11 share an amino-terminal transcription repression domain, which is required for the growth-suppressive effect of KLF11 [57]. Defects in Klf11 are the cause of maturity-onset diabetes of the young, type 7 [58]. Recently, it has been reported that Klf10 mRNA expression is regulated by CLOCK/BMAL1 heterodimers and that KLF10 itself suppresses lipogenic gene expression [59]. In Klf10 knockout mice, it has been reported that male $\mathrm{Klfl}^{-/-}$ mice displayed postprandial and fasting hyperglycemia, whereas $\mathrm{KlflO}^{-/-}$females were normoglycemic but displayed higher plasma triglyceride concentrations 
[59]. In addition, it has been demonstrated that glucose stimulation induces Klf10 mRNA expression in Rat-1 fibroblasts [60]. We have found that ChREBP directly regulates Klf10 mRNA expression in rat primary hepatocytes through binding to the carbohydrate response element (ChoRE), located between -125 bp and -108 bp in the rat Klf10 promoter [14]. Interestingly, adenoviral overexpression of KLF10 partly inhibits the glucose induction of ChREBP target genes in primary hepatocytes [14]. These data suggest that a feedback loop system between ChREBP and KLF10 may be involved in the regulation of the lipogenic pathway and in the development of metabolic syndrome (Fig. 4).

\subsubsection{PPARa}

PPAR $\alpha$ is an important regulator of genes involved in $\beta$-oxidation of acyl-CoA in liver [61]. Formerly, it has been reported that glucose suppresses Ppara mRNA levels in pancreatic $\beta$-cells [62]. Moreover, overexpression of ChREBP suppresses Ppara mRNA levels in pancreatic $\beta$-cells [62]. We also confirmed these data in brown adipocytes (our unpublished results). Crosstalk between ChREBP and PPAR $\alpha$ may have important roles in lipid metabolism, especially in liver and brown adipose tissues.

\subsubsection{Hormones, hormone receptor and its modulator Glucagon and Glucagon receptor}

cAMP-dependent protein kinase (PKA) phosphorylates ChREBP protein and inactivates ChREBP- $\alpha$ transactivity through inhibition of nuclear translocation and DNA binding to ChoRE in its target genes' promoters (Fig. 4) [24]. Glucagon increases the intracellular cAMP concentration and activates PKA [64]. Accordingly, glucagon negatively regulates ChREBP transactivity. By contrast, whether ChREBP affects glucagon mRNA has yet to be determined. Further investigation is required to clarify the crosstalk between glucagon and ChREBP in pancreatic $\alpha$-cells.

The glucagon receptor (GCGR) is essential for maintaining glucose homeostasis in the liver and for stimulating insulin secretion in pancreatic $\beta$-cells [64]. Glucose induces rat Gcgr mRNA expression; however, the precise mechanism remains unknown $[64,65]$. The G-box has previously been reported to be responsible for glucose regulation of Gcgr mRNA expression [65]. In the rat Gcgr promoter, a putative ChoRE (-554 bp/-538 bp) is localized near the G-box $(-543 \mathrm{bp} /-529 \mathrm{bp})[16,65]$. In rat INS-1E insulinoma cells, deletion studies of the rat Gcgr promoter showed that ChoRE is a minimal glucose response element
[16]. Furthermore, glucagon partly suppresses the glucose-induced expression of Gcgr mRNA [16]. Thus, ChREBP directly regulates rat $G c g r$ mRNA expression in INS-1E cells. In addition, a negative feedback loop between ChREBP and GCGR may further contribute to the regulation of glucose-induced gene expression.

The plasma glucagon concentration is normally elevated in the fasted state and suppressed in the fed state; however, in diabetic conditions the plasma glucagon concentration is elevated in both the fasted and fed states [64]. In the pathogenesis of type 2 diabetes mellitus, the constant abnormal elevation in both GCGR and plasma glucagon concentration may contribute to both fasting and postprandial hyperglycemia.

\section{Fibroblast growth factor 21 (FGF21)}

During fasting, FGF21 is induced and in turn increases fatty acid $\beta$-oxidation and plasma ketone bodies to supply fuels $[67,68]$. Recently, it has been reported that FGF21 is increased in obesity, and administration of FGF21 lowers plasma glucose levels and body weight in obesity $[67,68]$. These findings suggest that FGF21 has an important role in the development of metabolic syndrome [67, 68]. Moreover, it has been reported that insulin induces FGF21 in muscle [69]. We hypothesized that glucose also regulates $F g f 21$ mRNA expression. First, we identified that glucose induces Fgf 21 mRNA expression through ChREBP activation, and that the ChoRE is located between -74 and -52 bp in the rat $F g f 21$ promoter [15]. ChoRE in the mouse $F g f 21$ promoter is similar to the one in the rat $F g f 21$ promoter. In addition, in accordance with our data, adenoviral overexpression of ChREBP in liver induces Fgf 21 mRNA [70]. Thus, ChREBP increases Fgf 21 mRNA in rats and mice. However, there is controversy regarding its role in humans. Although ChREBP induces $F g f 21$ mRNA expression in human HepG2 cells [71], its ChoRE is composed of two E-boxes separated by $8 \mathrm{bp}$ instead of $5 \mathrm{bp}$ [71]. Together with the study demonstrating that 5 bp between the two E-boxes is essential [6], the human ChoRE does not seem to be functional. Accordingly, further investigation will be needed to identify a functional ChoRE in the human FGF21 promoter.

\section{The role of ChREBP in liver, pancreatic islets and adipose tissues}

\subsection{The physiological role of ChREBP in liver}

Carbohydrate response element binding protein 
(ChREBP) has an important role in hepatic de novo lipogenesis and in the development of metabolic syndrome. A study on Chrebp knockout mice demonstrated that ChREBP regulates $50 \%$ of hepatic de novo lipogenesis [2]. Normal diet-fed ChREBP knockout mice show moderate glucose intolerance compared with normal diet-fed wild-type mice [2]. Moreover, Chrebp ${ }^{-/-}$livers are in a hypoenergetic state, because Chrebp $p^{-/-}$liver has a near global reduction in hepatic fluxes with the exception of increased pyruvate oxidation [72]. Our group as well as other groups have independently reported that gene deletion of Chrebp or shRNA-based knockdown of Chrebp mRNA in ob/ob mice improves obesity, fatty liver, and glucose intolerance $[49,73]$. Similarly, adenoviral delivery of dnMlx, which antagonizes ChREBP transactivity, reduced hepatic triglyceride content and improved glucose intolerance by inhibiting the expression of G6pc and Elovl6 mRNA in addition to lipogenic enzymes [12]. Moreover, it has been reported that atorvastatin, a strong $\mathrm{HMG}-\mathrm{CoA}$ reductase inhibitor, prevents ChREBP activation and steatosis in fructose-fed rats by activating protein kinase A [74]. Thus, suppression of ChREBP will be beneficial for hepatic steatosis and glucose intolerance accompanied with hyperinsulinemia.

By contrast, it has been reported that adenoviral delivery of Chrebp cDNA into liver of high-fat diet-fed C57BL6/J mice causes fatty liver changes and improves glucose tolerance compared with control mice [70]. In addition, Chrebp mRNA expression decreases during the development of non-alcoholic steatohepatitis [70]. A couple of groups have reported that in adolescents with impaired glucose tolerance or T2DM (type 2 diabetes mellitus), the expression of ChREBP was increased in the liver [75, 76]. Similarly, hepatic Chrebp mRNA levels in obese subjects were much higher than in lean subjects [77]. Our preliminary study is compatible with these studies. We found that a transient increase in Chrebpb mRNA levels precedes glucose intolerance and fatty liver in C57BL6/J mice fed a high carbohydrate, high-fat diet (unpublished results). Moreover, the hepatic Chrebp mRNA levels in ob/ob mice were much higher than those in wild-type mice. However, the hepatic Chrebp mRNA levels in streptozotocin injected insulin deficient mice were similar to those in control mice (our unpublished results). These findings suggest that ChREBP expression levels and transactivity depend on plasma insulin concentration. Therefore, ChREBP activation may be involved in the early stage of metabolic syndrome, and ChREBP transactivity is probably rather suppressed during the late stage of metabolic syndrome with severe insulin resistance and insufficient insulin secretion.

\subsection{The physiological role of ChREBP in pancreatic islets}

An early study suggested that glucose induces $P k l r$ mRNA expression in INS-1E cells, a rat pancreatic insulinoma cell line [78]. After the identification of ChREBP, it has been reported that ChREBP rather than USF2 (upstream stimulatory factor 2) regulates the glucose stimulation of the endogenous L-pyruvate kinase expression in INS-1E cells [79]. Consistent with these data, we confirmed that glucose stimulates the expression of ChREBP target genes in INS-1E cells [39]. Thus, glucose induces ChREBP target genes through ChREBP activation in pancreatic insulin-producing cells. Furthermore, glucose is known to increase $\beta$-cell proliferation in vitro and in vivo [80]. Recently, it has been reported that in tumor cells, ChREBP plays a key role both in redirecting glucose metabolism to anabolic pathways and suppressing p53 activity [81]. It has also been reported that suppression of ChREBP reduces glucose-induced pancreatic $\beta$-cell proliferation as well as mRNA levels of cell cycle regulators such as $C c n d 2$, $C c n a$, and Ccne [80]. Thus, ChREBP is required for glucose-stimulated pancreatic $\beta$-cell proliferation. Whether overexpression of ChREBP causes glucotoxicity is currently not clear $[79,82]$. A former study reported that ChREBP overexpression did not affect glucose-stimulated insulin secretion in INS-1 cells [79]. Contrary to these data, it has been reported that adenoviral delivery of constitutively active ChREBP into pancreatic islets caused diabetes [82]. In this study, they used the rat insulin promoter for adenovirus construction, and they did not exclude problematic issues similar to the case of RIP transgenic mice [83]. Further investigation will be needed to determine whether overexpression of ChREBP affects insulin secretion and glucose toxicity. Finally, it has been reported that activation of ChREBP leads to increased pancreatic $\beta$-cell differentiation in rats [84]. When rat embryonic pancreases were cultured in the presence of glucose or xylitol, the mRNA levels of ChREBP target genes were induced and $\beta$-cell differentiation was enhanced. In this experiment, they used xylitol as a source of X5P [84]. However, unlike in hepatocytes, xylitol is difficult to convert into X5P in pancreatic islet cells, because of the much lower expression of xylulokinase [39]. It would be interest- 
ing to determine whether xylulokinase levels change during the development of the endocrine pancreas.

Recently, Leclerc et al. proposed a unique mechanism of ChREBP activation in pancreatic $\beta$-cells [85]. ChREBP interacts with sorcin, a penta-EF-hand $\mathrm{Ca}^{2+}$ binding protein that is localized in the cytosol at low glucose concentrations [85]. Interestingly, in sorcinsilenced $\beta$-cells, ChREBP is constitutively present in the nucleus [85]. Whether a similar ChREBP regulation mechanism exists in other inducible tissues such as muscle, in which ChREBP is abundantly expressed, would be interesting to investigate.

\subsection{The physiological role of ChREBP in adipose tissues}

Both ChREBP- $\alpha$ and ChREBP- $\beta$ are abundantly expressed in white and brown adipose tissues [2, 25]. Chrebp mRNA expression is increased during differentiation of 3T3-L1 cells [86]. Similarly, ChREBP is increased during differentiation of human omental and subcutaneous preadipocytes [77]. In adolescents with impaired glucose tolerance or T2DM, the expression of Chrebp is decreased in adipose tissue but increased in the liver [75]. It has been reported that decreased expression of Chrebp causes a decrease in peripheral glucose uptake and worsens insulin resistance through decreased expression of glucose transporter type 4 (Glut4) mRNA $[25,76]$. The finding of a novel ChREBP isoform is significant and fascinating. However, there are still some open questions. De novo lipogenesis in white adipose tissue and liver are not compatible with the expression levels of ChREBP in these tissues [25, 87]. Adenoviral delivery of shRNA against Chrebp mRNA into liver protects from fatty liver in ob/ob mice, which is in accordance with our data on ob/ob Chrebp $p^{-/-}$double knockout mice $[49,73]$. Moreover, we reported that adenoviral overexpression of dnMlx causes a decrease in hepatic triglyceride content [12]. In addition, we checked the Chrebpa and Chrebpb mRNA levels in liver, white adipose tissue, and brown adipose tissue, and found that Chrebph mRNA levels in liver and brown adipose tissues are much higher than in white adipose tissues (our unpublished results). Many groups have reported that triglycerides produced in liver are transferred to white adipose tissue, and that de novo lipogenesis in white adipose tissue is much lower than in liver [87, 88]. By contrast, de novo lipogenesis in brown adipose tissue is much higher than in white adipose tissue, which is consistent with the Chrebp mRNA levels [2, 87, 88]. Thus, to elucidate the specific role of ChREBP in adipose tissues, it is essential to identify whether it regulates the expression of adipokines such as leptin and adiponectin in white adipose tissue. As with de novo lipogenesis, the role of ChREBP in brown adipose tissues is more important. Investigating the role of ChREBP and PPAR $\alpha$ in brown adipocyte lipogenesis revealed that ChREBP and PPAR $\alpha$ constitute a feedback loop in brown adipose tissue (our unpublished results). The role of ChREBP in brown adipose tissue will be further investigated.

Finally, although overexpression of ChREBP or ChREBP activation in adipose tissues appears to be beneficial for treatment of T2DM, I think that overexpression of ChREBP in adipose tissues will only transiently normalize blood glucose and will consequently worsen obesity and insulin resistance. Obesity causes several harmful problems such as gonarthrosis, sleep apnea, dyslipidemia, hypertension, and glucose intolerance. We should keep in mind that to normalize not only glycemic control but also body weight, diet and exercise therapy is essential for the treatment of obesity related T2DM. Normalizing only blood glucose levels without reducing body weight and visceral fat mass is not sufficient for the treatment of obese patients with T2DM.

\section{Concluding remarks}

ChREBP target genes are involved in the pathways of glucose and lipid metabolism, circadian rhythm, and hormone/receptors, suggesting that ChREBP is involved in the development of metabolic syndrome. Therefore, understanding the mechanisms of ChREBP regulation will provide important insights for the treatment of obesity related diabetes mellitus. Moreover, it is important to identify the tissue-specific roles of ChREBP in liver, pancreatic islets, and adipose tissues. In particular, the following questions are of interest:

1. The differences in Chrebp mRNA levels in lean and obese subjects between white adipose tissue and liver:

In diabetic and obese subjects compared with lean subjects, changes in Chrebp mRNA levels in liver are different from those in adipose tissues.

What is the reason(s) for the differences in ChREBP regulation between liver and adipose tissues?

2.The adipocyte-specific function of ChREBP:

Does Chrebp regulate adipokines such as leptin, adi- 
ponectin and MCP-1?

3. The role of ChREBP in pancreatic $\alpha$-cells:

Does ChREBP or a paralogue of ChREBP known as MondoA regulate Glucagon mRNA expression through a mechanism similar to that of glucagon receptor?

Clarifying the role of ChREBP in several tissues will be beneficial for understanding the pathology of metabolic syndrome and for the treatment of obesity and its related metabolic disorders such as diabetes mellitus, non-alcoholic steatohepatitis and dyslipidemia.

\section{Acknowledgements}

The author would like to thank Professor Kosaku Uyeda for his guidance and interest. This work was supported in part by a Grant-in-Aid for Scientific Research from the Japan Society for the Promotion of Science, the Gifu University Graduate School of Medicine, the Kao Research Council for the Study of Healthcare Science, the Daiwa Securities Health Foundation, the Tsufu Zaidan, and the Japanese Diabetes Foundation.

\section{References}

1. Yamashita H, Takenoshita M, Sakurai M, Bruick RK, Henzel WJ, et al. (2001) A glucose-responsive transcription factor that regulates carbohydrate metabolism in the liver. Proc Natl Acad Sci U S A 98: 9116-9121.

2. Iizuka $\mathrm{K}$, Bruick RK, Liang G, Horton JD, Uyeda $\mathrm{K}$ (2004) Deficiency of carbohydrate response elementbinding protein (ChREBP) reduces lipogenesis as well as glycolysis. Proc Natl Acad Sci U S A 101: 72817286.

3. Cairo S, Merla G, Urbinati F, Ballabio A, Reymond A (2001) WBSCR14, a gene mapping to the Williams-Beuren syndrome deleted region, is a new member of the Mlx transcription factor network. Hum Mol Genet 10: 617-627.

4. Stoeckman AK, Ma L, Towle HC (2004) Mlx is the functional heteromeric partner of the carbohydrate response element-binding protein in glucose regulation of lipogenic enzyme genes. J Biol Chem 279: 1566215669.

5. Ishii S, Iizuka K, Miller BC, Uyeda K (2004) Carbohydrate response element binding protein directly promotes lipogenic enzyme gene transcription. Proc Natl Acad Sci U S A 101: 15597-15602.

6. Shih HM, Liu Z, Towle HC (1995) Two CACGTG motifs with proper spacing dictate the carbohydrate regulation of hepatic gene transcription. J Biol Chem 270: 21991-21997.

7. Jeong YS, Kim D, Lee YS, Kim HJ, Han JY, et al. (2011) Integrated expression profiling and genome-wide analysis of ChREBP targets reveals the dual role for ChREBP in glucose-regulated gene expression. PLoS One 6: e22544.

8. Ma L, Robinson LN, Towle HC (2006) ChREBP*Mlx is the principal mediator of glucose-induced gene expression in the liver. J Biol Chem 281: 28721-28730.

9. Iizuka K, Horikawa Y (2008) ChREBP: a glucose-activated transcription factor involved in the development of metabolic syndrome. Endocr J 55: 617-624.
10. Pedersen KB, Zhang P, Doumen C, Charbonnet M, Lu D, et al. (2007) The promoter for the gene encoding the catalytic subunit of rat glucose-6-phosphatase contains two distinct glucose-responsive regions. Am J Physiol Endocrinol Metab 292: E788-801.

11. Dubuquoy C, Robichon C, Lasnier F, Langlois C, Dugail I, et al. (2011) Distinct regulation of adiponutrin/PNPLA3 gene expression by the transcription factors ChREBP and SREBP1c in mouse and human hepatocytes. J Hepatol 55: 145-53.

12. Iizuka K, Takeda J, Horikawa Y (2009) Hepatic overexpression of dominant negative Mlx improves metabolic profile in diabetes-prone C57BL/6J mice. Biochem Biophys Res Commun 379: 499-504.

13. Iizuka K, Horikawa Y (2008) Regulation of lipogenesis via BHLHB2/DEC1 and ChREBP feedback looping. Biochem Biophys Res Commun 374: 95-100.

14. Iizuka K, Takeda J, Horikawa Y (2011) Krüppel-like factor-10 is directly regulated by carbohydrate response element-binding protein in rat primary hepatocytes. Biochem Biophys Res Commun 412: 638-643.

15. Iizuka K, Takeda J, Horikawa Y (2009) Glucose induces FGF21 mRNA expression through ChREBP activation in rat hepatocytes. FEBS Lett 583: 2882-2886.

16. Iizuka K, Tomita R, Takeda J, Horikawa Y (2012) Rat glucagon receptor mRNA is directly regulated by glucose through transactivation of the carbohydrate response element binding protein. Biochem Biophys Res Commun 417: 1107-1112.

17. Noriega LG, Feige JN, Canto C, Yamamoto H, Yu J, et al. (2011) CREB and ChREBP oppositely regulate SIRT1 expression in response to energy availability. EMBO Rep 12: 1069-1076.

18. Pashkov V, Huang J, Parameswara VK, Kedzierski W, Kurrasch DM, et al. (2011) Regulator of G protein signaling (RGS16) inhibits hepatic fatty acid oxidation in a carbohydrate response element-binding protein (ChREBP)-dependent manner. J Biol Chem 286: 
15116-15125.

19. da Silva Xavier G, Sun G, Qian Q, Rutter GA, Leclerc I (2010) ChREBP regulates Pdx-1 and other glucose-sensitive genes in pancreatic $\beta$-cells. Biochem Biophys Res Commun 402: 252-257.

20. Noordeen NA, Khera TK, Sun G, Longbottom ER, Pullen TJ, et al. (2010) Carbohydrate-responsive element-binding protein (ChREBP) is a negative regulator of ARNT/HIF-1beta gene expression in pancreatic islet beta-cells. Diabetes 59: 153-160.

21. Cha-Molstad H, Saxena G, Chen J, Shalev A (2009) Glucose-stimulated expression of Txnip is mediated by carbohydrate response element-binding protein, p300, and histone $\mathrm{H} 4$ acetylation in pancreatic beta cells. $J$ Biol Chem 284: 16898-16905.

22. Kawaguchi T, Osatomi K, Yamashita H, Kabashima T, Uyeda K (2002) Mechanism for fatty acid "sparing" effect on glucose-induced transcription: regulation of carbohydrate-responsive element-binding protein by AMP-activated protein kinase. $J$ Biol Chem 277: 38293835.

23. Kabashima T, Kawaguchi T, Wadzinski BE, Uyeda K (2003) Xylulose 5-phosphate mediates glucose-induced lipogenesis by xylulose 5-phosphate-activated protein phosphatase in rat liver. Proc Natl Acad Sci U S A 100: 5107-5112.

24. Kawaguchi T, Takenoshita M, Kabashima T, Uyeda K (2001) Glucose and cAMP regulate the L-type pyruvate kinase gene by phosphorylation/dephosphorylation of the carbohydrate response element binding protein. Proc Natl Acad Sci U S A 98:13710-13715.

25. Herman MA, Peroni OD, Villoria J, Schön MR, Abumrad NA, et al. (2012) A novel ChREBP isoform in adipose tissue regulates systemic glucose metabolism. Nature 484: 333-338.

26. Tsatsos NG, Towle HC (2006) Glucose activation of ChREBP in hepatocytes occurs via a two-step mechanism. Biochem Biophys Res Commun 340: 449-456.

27. Burke SJ, Collier JJ, Scott DK (2009) cAMP prevents glucose-mediated modifications of histone $\mathrm{H} 3$ and recruitment of the RNA polymerase II holoenzyme to the L-PK gene promoter. J Mol Biol 392: 578-588.

28. Burke SJ, Collier JJ, Scott DK (2009) cAMP opposes the glucose-mediated induction of the L-PK gene by preventing the recruitment of a complex containing ChREBP, HNF4alpha, and CBP. FASEB $J$ 23: 28552865.

29. Bricambert J, Miranda J, Benhamed F, Girard J, Postic C, Dentin R (2010) Salt-inducible kinase 2 links transcriptional coactivator p300 phosphorylation to the prevention of ChREBP-dependent hepatic steatosis in mice. J Clin Invest 120: 4316-4331.

30. Zeidan Q, Hart GW (2010) The intersections between O-GlcNAcylation and phosphorylation: implications for multiple signaling pathways. J Cell Sci 123: 13-22.
31. Guinez C, Filhoulaud G, Rayah-Benhamed F, Marmier S, Dubuquoy C, et al. (2011) O-GlcNAcylation increases ChREBP protein content and transcriptional activity in the liver. Diabetes 60: 1399-1413.

32. Sakiyama H, Fujiwara N, Noguchi T, Eguchi H, Yoshihara D, et al. (2010) The role of O-linked GlcNAc modification on the glucose response of ChREBP. Biochem Biophys Res Commun 402: 784-789.

33. Ido-Kitamura Y, Sasaki T, Kobayashi M, Kim HJ, Lee YS, et al. (2012) Hepatic FoxO1 integrates glucose utilization and lipid synthesis through regulation of Chrebp O-glycosylation. PLoS One 7: e47231.

34. Li MV, Chang B, Imamura M, Poungvarin N, Chan L (2006) Glucose-dependent transcriptional regulation by an evolutionarily conserved glucose-sensing module. Diabetes 55: 1179-1189.

35. Davies MN, O'Callaghan BL, Towle HC (2010) Activation and repression of glucose-stimulated ChREBP requires the concerted action of multiple domains within the MondoA conserved region. $\mathrm{Am} \mathrm{J}$ Physiol Endocrinol Metab 299: E665-674.

36. Li MV, Chen W, Poungvarin N, Imamura M, Chan L (2008) Glucose-mediated transactivation of carbohydrate response element-binding protein requires cooperative actions from Mondo conserved regions and essential trans-acting factor 14-3-3. Mol Endocrinol 22: 1658-1672.

37. Sakiyama H, Wynn RM, Lee WR, Fukasawa M, Mizuguchi H (2008) Regulation of nuclear import/ export of carbohydrate response element-binding protein (ChREBP): interaction of an alpha-helix of ChREBP with the 14-3-3 proteins and regulation by phosphorylation. J Biol Chem 283: 24899-24908.

38. Ge Q, Nakagawa T, Wynn RM, Chook YM, Miller BC, et al. (2011) Importin-alpha protein binding to a nuclear localization signal of carbohydrate response elementbinding protein (ChREBP). J Biol Chem 286:2811928127.

39. Iizuka K, Wu W, Horikawa Y, Takeda J (2013) Role of glucose-6-phosphate and xylulose-5-phosphate in the regulation of glucose-stimulated gene expression in the pancreatic $\beta$ cell line, INS-1E. Endocr J 60:473-482.

40. Cha JY, Repa JJ (2007) The liver X receptor (LXR) and hepatic lipogenesis. The carbohydrate-response element-binding protein is a target gene of LXR. $J$ Biol Chem 282: 743-751.

41. Sirek AS, Liu L, Naples M, Adeli K, Ng DS, et al. (2009) Insulin stimulates the expression of carbohydrate response element binding protein (ChREBP) by attenuating the repressive effect of Pit-1, Oct-1/Oct-2, and Unc-86 homeodomain protein octamer transcription factor-1. Endocrinology 150: 3483-3492.

42. Hashimoto K, Ishida E, Matsumoto S, Okada S, Yamada M, et al. (2009) Carbohydrate response element binding protein gene expression is positively regulated by thy- 
roid hormone. Endocrinology 150: 3417-3424.

43. Towle HC, Kaytor EN, Shih HM (1997) Regulation of the expression of lipogenic enzyme genes by carbohydrate. Anпи Rev Nutr 17: 405-433.

44. Girard J, Ferré P, Foufelle F (1997) Mechanisms by which carbohydrates regulate expression of genes for glycolytic and lipogenic enzymes. Annu Rev Nutr 17: 325-352.

45. Li MV, Chen W, Harmancey RN, Nuotio-Antar AM, Imamura M, et al. (2010) Glucose-6-phosphate mediates activation of the carbohydrate responsive binding protein (ChREBP). Biochem Biophys Res Commun 395: 395-400.

46. Dentin R, Tomas-Cobos L, Foufelle F, Leopold J, Girard J, et al. (2012) Glucose 6-phosphate, rather than xylulose 5-phosphate, is required for the activation of ChREBP in response to glucose in the liver. $J$ Hepatol 56: 199-209.

47. Arden C, Tudhope SJ, Petrie JL, Al-Oanzi ZH, Cullen KS, et al. (2012) Fructose 2,6-bisphosphate is essential for glucose-regulated gene transcription of glucose-6phosphatase and other ChREBP target genes in hepatocytes. Biochem J 443: 111-123.

48. van Schaftingen E, Gerin I (2002) The glucose-6-phosphatase system. Biochem J 362: 513-532.

49. Iizuka K, Miller B, Uyeda K (2006) Deficiency of carbohydrate-activatedtranscription factor ChREBP prevents obesity and improves plasma glucose control in leptin-deficient (ob/ob) mice. Am J Physiol Endocrinol Metab 291: E358-E364.

50. Ko CH, Takahashi JS (2006) Molecular components of the mammalian circadian clock. Hum Mol Genet 15: R271-277.

51. Honma S, Kawamoto T, Takagi Y, Fujimoto K, Sato F, et al. (2002) Dec1 and Dec2 are regulators of the mammalian molecular clock. Nature 419:841-844.

52. Nakashima A, Kawamoto T, Honda KK, Ueshima T, Noshiro M, et al. (2008) DEC1 modulates the circadian phase of clock gene expression. Mol Cell Biol 28: 40804092.

53. Turek FW, Joshu C, Kohsaka A, Lin E, Ivanova G, et al. (2005) Obesity and metabolic syndrome in circadian Clock mutant mice. Science 308: 1043-1045.

54. Coletta DK, Balas B, Chavez AO, Baig M, Abdul-Ghani $\mathrm{M}$, et al. (2008) Effect of acute physiological hyperinsulinemia on gene expression in human Skeletal muscle in vivo. Am J Physiol Endocrinol Metab 294: E910-E917.

55. Cho Y, Noshiro M, Choi M, Morita K, Kawamoto T, et al. (2009) The basic helix-loop-helix proteins differentiated embryo chondrocyte (DEC) 1 and DEC2 function as corepressors of retinoid X receptors. Mol Pharmacol 76: 1360-1369.

56. Choi SM, Cho HJ, Cho H, Kim KH, Kim JB, et al. (2008) Stra13/DEC1 and DEC2 inhibit sterol regulatory element binding protein- $1 \mathrm{c}$ in a hypoxia-inducible fac- tor-dependent mechanism. Nucleic Acids Res 36: 63726385.

57. McConnell BB, Yang VW (2010) Mammalian Krüppellike factors in health and diseases. Physiol Rev 90: 1337-81.

58. Neve B, Fernandez-Zapico ME, Ashkenazi-Katalan V, Dina C, Hamid YH, et al. (2005) Role of transcription factor KLF11 and its diabetes-associated gene variants in pancreatic beta cell function. Proc Natl Acad Sci U S A 102: 4807-4812.

59. Guillaumond F, Gréchez-Cassiau A, Subramaniam M, Brangolo S, Peteri-Brünback B, et al. (2010) Kruppellike factor KLF10 is a link between the circadian clock and metabolism in liver. Mol Cell Biol 30: 3059-3070.

60. Hirota T, Okano T, Kokame K, Shirotani-Ikejima H, Miyata T, et al. (2002) Glucose down-regulates Per1 and Per2 mRNA levels and induces circadian gene expression in cultured Rat-1 fibroblasts. $J$ Biol Chem 277: 44244-44251.

61. Desvergne B, Wahli W (1999) Peroxisome proliferator-activated receptors: nuclear control of metabolism. Endocr Rev 20: 649-688.

62. Ravnskjaer K, Boergesen M, Dalgaard LT, Mandrup S (2006) Glucose-induced repression of PPARalpha gene expression in pancreatic beta-cells involves PP2A activation and AMPK inactivation. $J$ Mol Endocrinol 36: 289-299.

63. Boergesen M, Poulsen Ll, Schmidt SF, Frigerio F, Maechler P, et al. (2011) ChREBP mediates glucose repression of peroxisome proliferator-activated receptor alpha expression in pancreatic beta-cells. $J$ Biol Chem 286:13214-13225.

64. Edgerton DS, Cherrington AD (2011) Glucagon as a critical factor in the pathology of diabetes. Diabetes 60: 377-380.

65. Portois L, Maget B, Tastenoy M, Perret J, Svoboda M (1999) Identification of a glucose response element in the promoter of the rat glucagon receptor gene. $J$ Biol Chem 274: 8181-8190.

66. Unger RH, Cherrington AD (2012) Glucagonocentric restructuring of diabetes: a pathophysiologic and therapeutic makeover. J Clin Invest 122: 4-12.

67. Potthoff MJ, Kliewer SA, Mangelsdorf DJ (2012) Endocrine fibroblast growth factors 15/19 and 21: from feast to famine. Genes Dev 26: 312-324.

68. Kharitonenkov A, Shiyanova TL, Koester A, Ford AM, Micanovic R, et al. (2005) FGF-21 as a novel metabolic regulator. J Clin Invest 115: 1627-1635.

69. Izumiya Y, Bina HA, Ouchi N, Akasaki Y, Kharitonenkov A, et al. (2008) FGF21 is an Akt-regulated myokine. FEBS Lett 582: 3805-3810.

70. Benhamed F, Denechaud PD, Lemoine M, Robichon C, Moldes M, et al. (2012) The lipogenic transcription factor ChREBP dissociates hepatic steatosis from insulin resistance in mice and humans. $J$ Clin Invest 122: 2176- 
2194.

71. Uebanso T, Taketani Y, Yamamoto H, Amo K, Ominami $\mathrm{H}$, et al. (2011) Paradoxical regulation of human FGF21 by both fasting and feeding signals: is FGF21 a nutritional adaptation factor? PLoS One 6: e22976.

72. Burgess SC, Iizuka $\mathrm{K}$, Jeoung $\mathrm{NH}$, Harris RA, Kashiwaya Y, et al. (2008) Carbohydrate-response element-binding protein deletion alters substrate utilization producing an energy-deficient liver. $\mathrm{J}$ Biol Chem 283: 1670-1678.

73. Dentin R, Benhamed F, Hainault I, Fauveau V, Foufelle F, et al. (2006) Liver-specific inhibition of ChREBP improves hepatic steatosis and insulin resistance in ob/ ob mice. Diabetes 55: 2159-2170.

74. Rodríguez-Calvo R, Barroso E, Serrano L, Coll T, Sánchez RM, et al. (2009) Atorvastatin prevents carbohydrate response element binding protein activation in the fructose-fed rat by activating protein kinase A. Hepatology 49: 106-115.

75. Kursawe R, Caprio S, Giannini C, Narayan D, Lin A, et al. (2013) Decreased Transcription of ChREBP- $\alpha / \beta$ Isoforms in Abdominal Subcutaneous Adipose Tissue of Obese Adolescents With Prediabetes or Early Type 2 Diabetes: Associations With Insulin Resistance and Hyperglycemia. Diabetes 62: 837-844.

76. Eissing L, Scherer T, Tödter K, Knippschild U, Greve JW, et al. (2013) De novo lipogenesis in human fat and liver is linked to ChREBP- $\beta$ and metabolic health. Nat Commun 4:1528.

77. Hurtado del Pozo C, Vesperinas-García G, Rubio MÁ, Corripio-Sánchez R, Torres-García AJ, et al. (2011) ChREBP expression in the liver, adipose tissue and differentiated preadipocytes in human obesity. Biochim Biophys Acta 1811: 1194-200.

78. Roche E, Assimacopoulos-Jeannet F, Witters LA, Perruchoud B, Yaney G, et al. (1997) Induction by glucose of genes coding for glycolytic enzymes in a pancreatic beta-cell line (INS-1). J Biol Chem 272: 30913098.

79. Wang H, Kouri G, Wollheim CB (2005) ER stress and SREBP-1 activation are implicated in beta-cell glucoli- potoxicity. J Cell Sci 118: 3905-3915.

80. Metukuri MR, Zhang P, Basantani MK, Chin C, Stamateris RE, et al. (2012) ChREBP Mediates GlucoseStimulated Pancreatic $\beta$-Cell Proliferation. Diabetes 61: 2004-2015.

81. Tong X, Zhao F, Mancuso A, Gruber JJ, Thompson CB (2009) The glucose-responsive transcription factor ChREBP contributes to glucose-dependent anabolic synthesis and cell proliferation. Proc Natl Acad Sci US A 106: 21660-21665.

82. Poungvarin N, Lee JK, Yechoor VK, Li MV, Assavapokee T, et al. (2012) Carbohydrate response element-binding protein (ChREBP) plays a pivotal role in beta cell glucotoxicity. Diabetologia 55: 1783-1796.

83. Lee JY, Ristow M, Lin X, White MF, Magnuson MA, et al. (2006) RIP-Cre revisited, evidence for impairments of pancreatic beta-cell function. J Biol Chem 281: 26492653.

84. Soggia A, Flosseau K, Ravassard P, Szinnai G, Scharfmann R, et al. (2012) Activation of the transcription factor carbohydrate-responsive element-binding protein by glucose leads to increased pancreatic beta cell differentiation in rats. Diabetologia 55: 2713-2722.

85. Leclerc I, Rutter GA, Meur G, Noordeen N (2012) Roles of $\mathrm{Ca} 2+$ ions in the control of ChREBP nuclear translocation. J Endocrinol 213: 115-122.

86. He Z, Jiang T, Wang Z, Levi M, Li J (2004) Modulation of carbohydrate response element-binding protein gene expression in 3T3-L1 adipocytes and rat adipose tissue. Am J Physiol Endocrinol Metab 287: E424-430.

87. Trayhurn P (1981) Fatty acid synthesis in mouse brown adipose tissue the influence of environmental temperature on the proportion of whole-body fatty acid synthesis in brown adipose tissue and the liver. Biochim Biophys Acta 664: 549-560.

88. Agius L, Rolls BJ, Rowe EA, Williamson DH (1983) High-energy diets produce different effects on fatty acid synthesis in brown adipose tissue, white adipose tissue and liver in the rat. Biochim Biophys Acta 750: 383387. 